\title{
Propagation of elastic pressure waves in a beam window
}

\author{
T. R. Davenne* and P. Loveridge \\ Rutherford Appleton Laboratory, Chilton, Oxon OX11 0QX, United Kingdom
}

(Received 27 May 2016; published 12 September 2016)

\begin{abstract}
As particle accelerator beam power increases, stress on beam windows and targets increases. Many simulations are carried out to model the dynamic stresses that are induced in these critical components by near instantaneous beam heating. However while it is often easy to obtain simulation results there are few analytical solutions available to check the accuracy of simulation techniques. We follow the strand of several authors over the years who have offered analytical solutions to the classic problem of radial stress waves in a beam window. Many of these significant contributions have still had niggling issues with regard to resolving peak stress and limitations on the applied initial heating condition. We formulate an analytical expression for the radial pressure waves based on a Green's function solution of Feynman's wave equation. A complete analysis of the problem demonstrates that a hypothesis that beam induced pressure waves are composed of a static and transient component is indeed correct. The analytical expression is shown to give stable bounded solutions with easily determined peak stress levels. Finally a comparison between analytical expression and finite element analysis of the problem yields some general guidelines that should be adhered to for achieving accurate stress wave simulations.
\end{abstract}

DOI: 10.1103/PhysRevAccelBeams.19.093501

\section{INTRODUCTION}

The interaction of a particle beam with a beam window or target causes heating of the material in a time much shorter than the time required for the material to physically expand. This results in higher stress levels than would occur if the material was heated by the same amount slowly and what is generally referred to as an inertial response manifested as the propagation of pressure waves through the material. In many high energy physics experiments involving accelerators and in neutron spallation sources beam windows are used to separate the particle accelerator vacuum from the target station atmosphere or to separate the target station atmosphere from a target cooling circuit. A beam window failure would mean closure of a facility for some considerable time and damage to critical components. As such it is important to understand the safe operating limits of these critical components. The peak induced mechanical stress in a beam window must be compared to the strength or fatigue limit of the material as part of good component design.

Finite element analysis (FEA) is often used to calculate this important value of peak beam induced stress. For reasons of achieving safe long term operation beam windows are typically designed to operate within the elastic regime so that in continued service any induced stress waves

\footnotetext{
*Corresponding author. tristan.davenne@stfc.ac.uk

Published by the American Physical Society under the terms of the Creative Commons Attribution 3.0 License. Further distribution of this work must maintain attribution to the author(s) and the published article's title, journal citation, and DOI.
}

only result in completely reversible elastic deformations of the window material. As such we can simulate the stress using a Hooke's law linear elastic model to represent the strength of the window material. Explicit hydro-codes such as Autodyn and LS-Dyna have also been used to simulate elastic and plastic stress wave propagation [1,2]. The forward marching solvers employed by these explicit codes also allow them to simulate shock waves where there are appreciable changes in density of the material. This capability is not required for the simulation of beam windows and targets which are designed to operate in the elastic regime.

There are certain rules which must be adhered to when simulating inertial effects in windows with finite element analysis. In order to highlight these rules we present an analytical expression for the one dimensional propagation of radial pressure waves in a disc and then compare this with an implicit FEA simulation. There have been several previous presentations of analytical solutions for the propagation of radial waves in discs over the years. The most famous is that of Sievers in 1974 [3] where he presents useful analytical expressions for the stress in a constrained disc resulting from instantaneous and ramped heating in a "top hat" profile (Fig. 1) represented by the following expressions.

$$
\begin{gathered}
T=T_{0} \quad \text { if } 0 \leq r \leq r_{0} \\
T=0 \quad \text { if } r_{0} \leq r \leq R
\end{gathered}
$$

In 2000 Montanez and Sievers [1] presented new validation of Sievers' original work showing good agreement between the hydro-code LS-Dyna and the expressions 


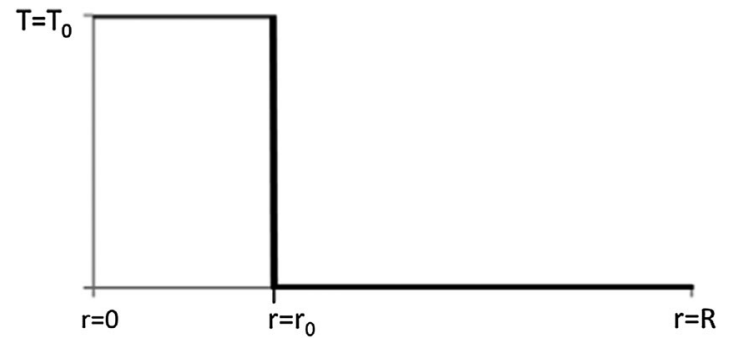

FIG. 1. "Top hat" initial condition as employed by Sievers et al.

from 1974. One observation of the presented results is that the peak stress predicted by the analytical solution does not agree with the finite element modeling, the sharp peaks in the analytical solution are put down to the discontinuity in initial condition and these are apparently smeared out in the numerical solution. Zheng et al. [4] published a solution for the radial stress waves in a disc subject to a smooth initial condition defined as follows and shown in Fig. 2.

$$
\begin{gathered}
T=T_{0} \quad \text { if } 0 \leq r \leq r_{0}-\delta \\
T=\frac{T_{0}}{2}\left[1-\sin \frac{\pi}{2 \delta}\left(r-r_{0}\right)\right] \quad \text { if } r_{0}-\delta \leq r \leq r_{0}+\delta \\
T=0 \quad \text { if } r_{0} \leq r \leq R
\end{gathered}
$$

We note their example results in significant stress spikes similar to those shown by Sievers. Here we present an analytical expression for the pressure in a beam window following sudden heating by a Gaussian beam defined as follows and shown in Fig. 3.

$$
T(r)=T_{0} e^{-\left(r^{2} / 2 \sigma^{2}\right)}
$$

We are interested in a Gaussian heating profile as many of the particle accelerator beams we work with are Gaussian in nature, however it is easily seen that the method presented can be used for other spatial heating functions. We first assume that the total pressure induced by the beam heating is comprised of a "quasistatic" component and a transient inertial component as follows

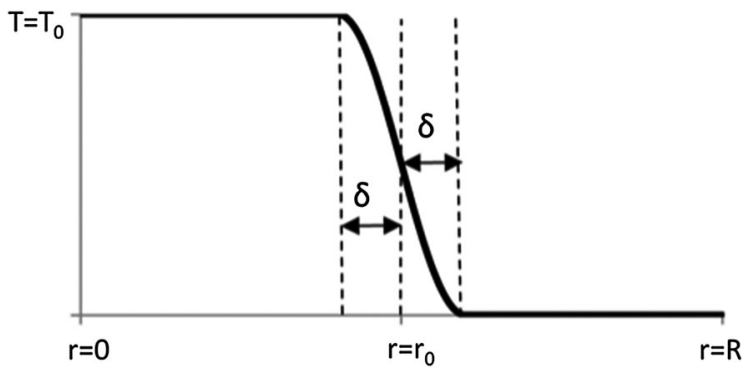

FIG. 2. Continuous initial condition as employed by Zheng et al.

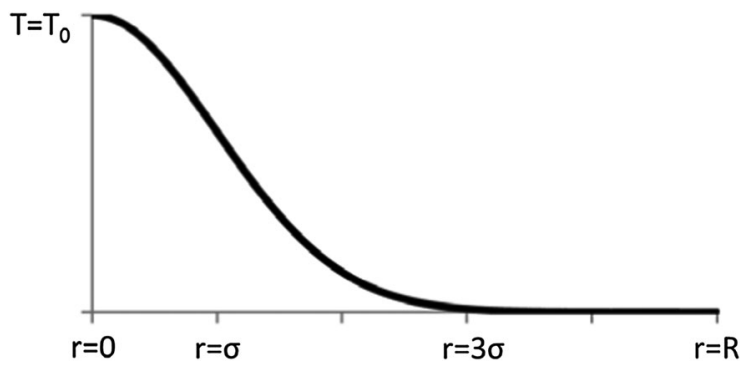

FIG. 3. Gaussian initial condition as used in the method presented here.

$$
P(r, t)=p_{s}(r)+p_{t}(r, t)
$$

where $P(r, t)$ is the total pressure, $p_{s}(r)$ is the quasistatic component of pressure and $p_{t}(r, t)$ is the transient component of pressure. We use some classical mechanics to derive the quasistatic component resulting from the imposed temperature profile. We refer to this as static as it is only reduced by thermal conduction typically taking place relatively slowly compared to the passage of radial waves. We then present a solution to the one dimensional radial wave equation as derived by Feynman [5] to obtain the transient component of pressure in the beam window. A test case is used to compare the final analytical expression with results from the finite element code ANSYS with a view to providing guidance for setting up FEA models of beam induced pressure waves.

\section{FORMULATION OF THE ANALYTICAL EXPRESSION}

We first determine the static pressure for the case of plane stress, the equivalent result for plane strain is presented later. Plane stress assumes no stress perpendicular to the radial direction akin to a foil or beam window. Plane strain assumes no axial deflection and is akin to an infinitely long cylinder, i.e., the problem addressed by Zheng [4]. The following plane stress expressions for radial stress, $\sigma_{r}$, and hoop stress, $\sigma_{\theta}$, [6] in a disc with temperature as a function of radius and with no deflection at $r=R$ (i.e., constrained at the outer radius) are used to determine the static pressure.

$$
\begin{aligned}
& \sigma_{r}=-E \alpha {\left[\frac{1}{r^{2}} \int_{0}^{r} T(r) \mathrm{rdr}+\frac{(1+\nu)}{(1-\nu)} \frac{1}{R^{2}} \int_{0}^{R} T(r) \mathrm{rdr}\right] } \\
& \sigma_{\theta}=-E \alpha\left[-\frac{1}{r^{2}} \int_{0}^{r} T(r) \mathrm{rdr}+\frac{(1+\nu)}{(1-\nu)} \frac{1}{R^{2}}\right. \\
&\left.\times \int_{0}^{R} T(r) \mathrm{rdr}+T(r)\right]
\end{aligned}
$$

Where $E$ is the Young's modulus, $\alpha$ is the thermal expansion coefficient, $v$ is Poisson's ratio and $R$ is the radius of the window. In the case of plane stress, pressure is directly related to stress in the following way and is the average of the two principal components (radial and hoop), i.e., 


$$
P=-\left(\frac{\sigma_{r}+\sigma_{\theta}}{2}\right)
$$

We substitute the Gaussian temperature profile [Eq. (6)] into Eqs. (8) and (9), then following integration of Eq. (10) we arrive at Eq. (11) for the static pressure as a function of radius.

$$
p_{s}(r)=E \alpha T_{0}\left[\frac{(1+\nu)}{(1-\nu)} \frac{\sigma^{2}}{R^{2}}\left\{1-e^{\left(-R^{2} / 2 \sigma^{2}\right)}\right\}+\frac{1}{2} e^{\left(-r^{2} / 2 \sigma^{2}\right)}\right]
$$

Now addressing the transient component we start with the radial wave equation as defined by Feynman

$$
\frac{1}{c^{2}} \frac{\partial^{2} p_{t}}{\partial t^{2}}=\frac{1}{r} \frac{\partial p_{t}}{\partial r}+\frac{\partial^{2} p_{t}}{\partial r^{2}}
$$

where $p_{t}$ is the transient component of pressure, and there is no variation in pressure with angle and $\mathrm{c}$ is the speed of sound in the material which for the plane stress problem is calculated as follows with $\rho$ representing density of the window material [7].

$$
c=\sqrt{\frac{E}{\rho\left(1-\nu^{2}\right)}}
$$

The following initial condition is used

$$
\frac{\partial p_{t}}{\partial t}(t=0)=0
$$

and in addition the Neumann boundary condition which implies no displacement at $r=R$ i.e., the outer rim of the window is constrained.

$$
\frac{\partial p_{t}}{\partial r}(r=R)=0
$$

According to Polyanin [8,9], the solution to Eq. (12) with the given initial and boundary conditions can be represented with the following integral,

$$
p_{t}(r, t)=\frac{\partial}{\partial t} \int_{0}^{R} p_{t}(\varepsilon, 0) G(r, \varepsilon, t) d \varepsilon
$$

where $G$ is a Green's function defined in the following way and $\varepsilon$ is an additional spatial variable with the same bounds as $r$, i.e., $0<\varepsilon<R$,

$$
\begin{aligned}
G(r, \varepsilon, t)= & \frac{2 t \varepsilon}{R^{2}}+\frac{2 \varepsilon}{c R} \sum_{n=1}^{\infty} \frac{1}{\lambda_{n} J_{0}^{2}\left(\lambda_{n}\right)} J_{0}\left(\lambda_{n} \frac{r}{R}\right) \\
& \times J_{0}\left(\lambda_{n} \frac{\varepsilon}{R}\right) \sin \left(\lambda_{n} \frac{c t}{R}\right)
\end{aligned}
$$

and $\lambda_{n}$ represents the roots of the Bessel function of order 1 , i.e., $J_{1}(\lambda)=0$. Noting that $\frac{\partial}{\partial t} \int_{0}^{R} p_{t}(\varepsilon, 0) G(r, \varepsilon, t) d \varepsilon=\int_{0}^{R} p_{t}(\varepsilon, 0) \frac{\partial}{\partial t} G(r, \varepsilon, t) d \varepsilon$

and also

$$
\begin{aligned}
\frac{\partial}{\partial t} G(r, \varepsilon, t)= & \frac{2 \varepsilon}{R^{2}}+\frac{2 \varepsilon}{R^{2}} \sum_{n=1}^{\infty} \frac{1}{J_{0}^{2}\left(\lambda_{n}\right)} J_{0}\left(\lambda_{n} \frac{r}{R}\right) \\
& \times J_{0}\left(\lambda_{n} \frac{\varepsilon}{R}\right) \cos \left(\lambda_{n} \frac{\mathrm{ct}}{R}\right)
\end{aligned}
$$

Then substituting Eq. (19) back into Eq. (18) we have the following general expression for any initial transient pressure profile

$$
\begin{aligned}
p_{t}(r, t)= & \frac{2 r}{R^{2}}+\frac{2}{R^{2}} \sum_{n=1}^{\infty} \frac{1}{J_{0}^{2}\left(\lambda_{n}\right)} J_{0}\left(\lambda_{n} \frac{r}{R}\right) \cos \left(\lambda_{n} \frac{c t}{R}\right) \\
& \times \int_{0}^{R} r p_{t}(r, 0) J_{0}\left(\lambda_{n} \frac{r}{R}\right) \mathrm{dr}
\end{aligned}
$$

Now we must turn our attention to the initial transient pressure profile that results from constant volume heating. We assume instantaneous heating of the window at $t=0$ such that the material has no time to expand during heating, i.e., constant volume heating. This is a reasonable assumption if the beam pulse duration is significantly shorter than the characteristic wave propagation time. Instantaneous heating results in a total pressure, $P$, that is purely the pressure due to constant volume heating denoted here as $P_{\mathrm{cv}}$. We then derive the transient component of pressure at $t=0$ using Eq. (7)

$$
p_{t}(r, t=0)=P_{\mathrm{cv}}(r)-p_{s}(r)
$$

where for the case of plane stress, it can be shown that constant volume heating results in the following pressure as a function of the temperature profile

$$
P_{\mathrm{cv}}(r)=\frac{E \alpha T(r)}{1-\nu}
$$

Substituting Eqs. (11) and (22) into (21) we arrive at the initial condition for the transient pressure resulting from Gaussian heating.

$$
\begin{aligned}
p_{t}(r, t=0)= & \frac{E \alpha T_{0}(1+\nu)}{1-\nu} \\
& \times\left[\frac{1}{2} e^{\left(-r^{2} / 2 \sigma^{2}\right)}-\frac{\sigma^{2}}{R^{2}}\left\{1-e^{\left(-R^{2} / 2 \sigma^{2}\right)}\right\}\right]
\end{aligned}
$$

Applying this into Eq. (20) gives us the following expression for the transient component of pressure in the disc as a result of the instantaneous Gaussian heating 


$$
\begin{aligned}
p_{t}(r, t)= & \frac{2}{R^{2}} \frac{E \alpha T_{0}(1+v)}{1-v}\left[\sum_{n=1}^{\infty} \frac{J_{0}\left(\lambda_{n} \frac{r}{R}\right)}{J_{0}^{2}\left(\lambda_{n}\right)} \cos \left(\lambda_{n} \frac{c t}{R}\right)\right. \\
& \times \int_{0}^{R} r\left[\frac{1}{2} e^{\left(-r^{2} / 2 \sigma^{2}\right)}-\frac{\sigma^{2}}{R^{2}}\left\{1-e^{\left(-R^{2} / 2 \sigma^{2}\right)}\right\}\right] \\
& \left.\times J_{0}\left(\lambda_{n} \frac{r}{R}\right) \mathrm{dr}\right]
\end{aligned}
$$

We now have the two terms of Eq. (7) and so based on our premise that the static and transient components of pressure make up the total pressure we have derived an analytical expression for the pressure in a beam window following instantaneous Gaussian heating. Equation (24) can be easily represented with a simple code to perform the required summation and numerical integration. A stable accurate solution is found with a modest number of terms.

At this point it is useful for the engineer of beam windows to note the relationships between the calculated pressure and stress. Examination of Eqs. (8) and (9) reveals that radial and hoop stress are equal at $r=0$ and that at this point $P=-\sigma_{r}$. We also find that $\mathrm{P}$ is a maximum at $r=0$. In other words the peak pressure occurring at the center of the disc also signifies the peak radial stress in the disc. Using the Von-Mises criteria for plane stress i.e., [6],

$$
\sigma_{\mathrm{VM}}=\sqrt{\left(\sigma_{r}^{2}+\sigma_{\theta}^{2}-\sigma_{r} \sigma_{\theta}\right)}
$$

Then at $r=0$ (where $\sigma_{r}=\sigma_{\theta}$ ), Eq. (25) simplifies to

$$
\sigma_{\mathrm{VM}}=\left|\sigma_{r}\right|=|P|
$$

For the case of plane strain we follow the same procedure as above, except that sound speed, constant volume pressure and static pressure must be calculated for the case of no axial deflection. For brevity the derivation of these terms is left to the reader.

$$
\begin{gathered}
c=\sqrt{\frac{E(1-\nu)}{\rho(1+\nu)(1-2 \nu)}} \\
P_{\mathrm{cv}}(r)=\frac{E \alpha T(r)}{(1-2 \nu)}
\end{gathered}
$$

For the static pressure we again refer to classic mechanics [6]. In plane strain the longitudinal stress component $\sigma_{z}$ is nonzero and so this time we have three components of stress as follows

$$
\begin{gathered}
\sigma_{r}=-\frac{\alpha E}{(1-\nu) r^{2}}\left[\frac{r^{2}}{R^{2}(1-2 \nu)} \int_{0}^{R} \mathrm{~T}(\mathrm{r}) \mathrm{rdr}+\int_{0}^{r} \mathrm{~T}(\mathrm{r}) \mathrm{rdr}\right] \\
\sigma_{\theta}=-\frac{\alpha E}{(1-\nu) r^{2}}\left[\frac{r^{2}}{R^{2}(1-2 \nu)} \int_{0}^{R} \mathrm{~T}(\mathrm{r}) \mathrm{rdr}\right. \\
\left.-\int_{0}^{r} \mathrm{~T}(\mathrm{r}) \mathrm{rdr}+\mathrm{T}(\mathrm{r}) \mathrm{r}^{2}\right]
\end{gathered}
$$

$$
\sigma_{z}=-\frac{\alpha E}{(1-\nu)}\left[\frac{2 \nu}{R^{2}(1-2 \nu)} \int_{0}^{R} \mathrm{~T}(\mathrm{r}) \mathrm{rdr}+\mathrm{T}(\mathrm{r})\right]
$$

All three principle components of stress must now be considered in the relation between pressure and stress

$$
p_{s}=-\frac{\left(\sigma_{r}+\sigma_{\theta}+\sigma_{z}\right)}{3}
$$

We substitute the Gaussian temperature profile shown in Eq. (6) into Eqs. (29), (30) and (31). Following integration of Eq. (32) we arrive at Eq. (33) for the static pressure as a function of radius.

$$
\begin{aligned}
p_{s}(r)= & \frac{2 E \alpha T_{0}}{3(1-\nu)} \\
& \times\left[\frac{\sigma^{2}(1+\nu)}{R^{2}(1-2 \nu)}\left\{1-e^{\left(-R^{2} / 2 \sigma^{2}\right)}\right\}+e^{\left(-r^{2} / 2 \sigma^{2}\right)}\right]
\end{aligned}
$$

Substituting Eqs. (33) and (28) into (21) we arrive at the initial condition for the transient pressure resulting from Gaussian heating.

$$
\begin{aligned}
p_{t}(r, t=0)= & \frac{E \alpha T_{0}(1+\nu)}{(1-\nu)(1-2 \nu)} \\
& \times\left[\frac{1}{3} e^{\left(-r^{2} / 2 \sigma^{2}\right)}-\frac{2 \sigma^{2}}{3 R^{2}}\left\{1-e^{\left(-R^{2} / 2 \sigma^{2}\right)}\right\}\right]
\end{aligned}
$$

Equations (33) and (34) can be combined in the same way as was done for the plane stress case in order to find the total pressure.

\section{COMPARISON WITH FINITE ELEMENT ANALYSIS}

We now consider a test case of a grade 5 titanium alloy beam window with the following parameters

Window radius, $R$

Young's modulus, $E$

Linear thermal expansion coefficient, $\alpha$

Poisson's ratio, $\nu$

Density, $\rho$

Gaussian temperature coefficient, $T_{0}$

Gaussian sigma, $\sigma$

$$
\begin{gathered}
0.05 \mathrm{~m} \\
114 \mathrm{GPa} \\
8.6 \times 10^{-6} \mathrm{~K}^{-1} \\
0.34 \\
4430 \mathrm{~kg} / \mathrm{m}^{3} \\
100 \mathrm{~K} \\
0.01 \mathrm{~m}
\end{gathered}
$$

ANSYS Mechanical was used [10] to complete a finite element analysis of the test case using plane elements with the option for plane-stress or plane-strain set as required. The finite element analysis uses an implicit solver and assumes a linear elastic material model based on Hooke's law. Transient structural time integration is employed to simulate the inertial effects. We note that in the following comparisons the plotted pressures are normalized by dividing by the initial pressure at $r=0$ as follows 


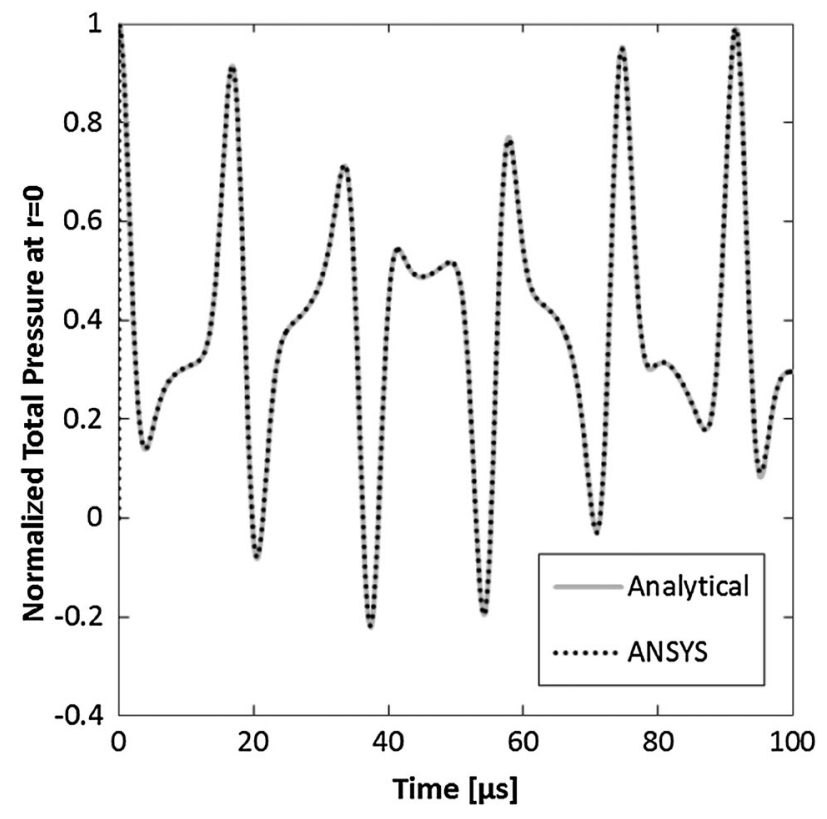

FIG. 4. Normalized total pressure at $r=0$ for the test case assuming plane stress.

$$
\text { normalized pressure }=\frac{\text { pressure }}{P_{\mathrm{cv}}(r=0)}
$$

Figures 4, 5 and 6 show the normalized total pressure at different radial positions demonstrating perfect agreement between the ANSYS simulation and the analytical expression presented here. Figure 7 shows the Von-Mises stress occurring in the window at $r=0$ as calculated by the expressions presented here and from ANSYS. In order to

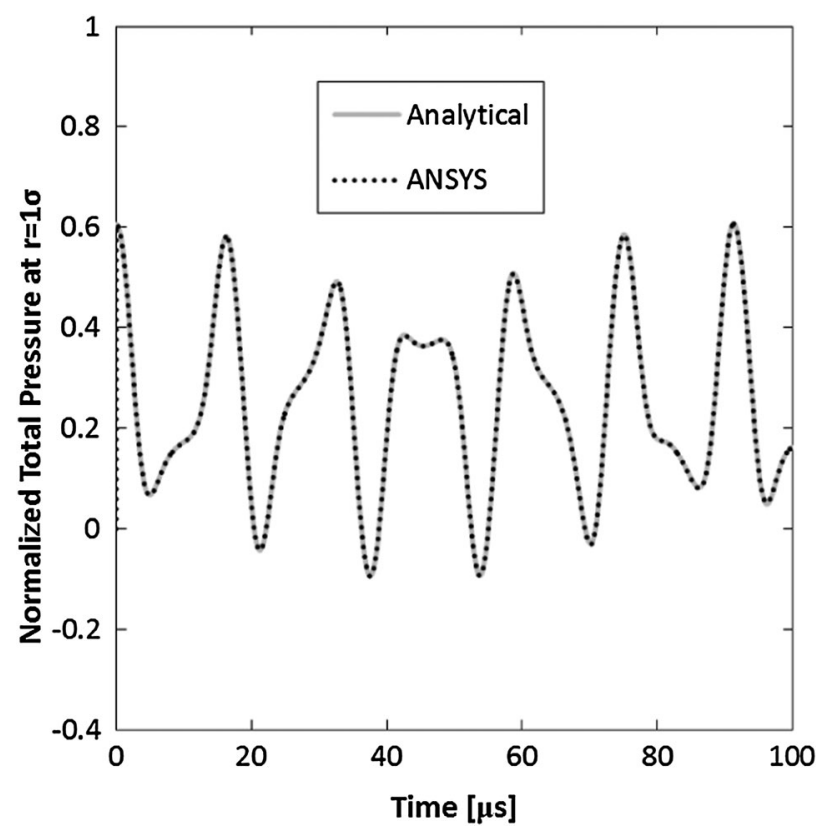

FIG. 5. Normalized total pressure at $r=\sigma$ for the test case assuming plane stress.

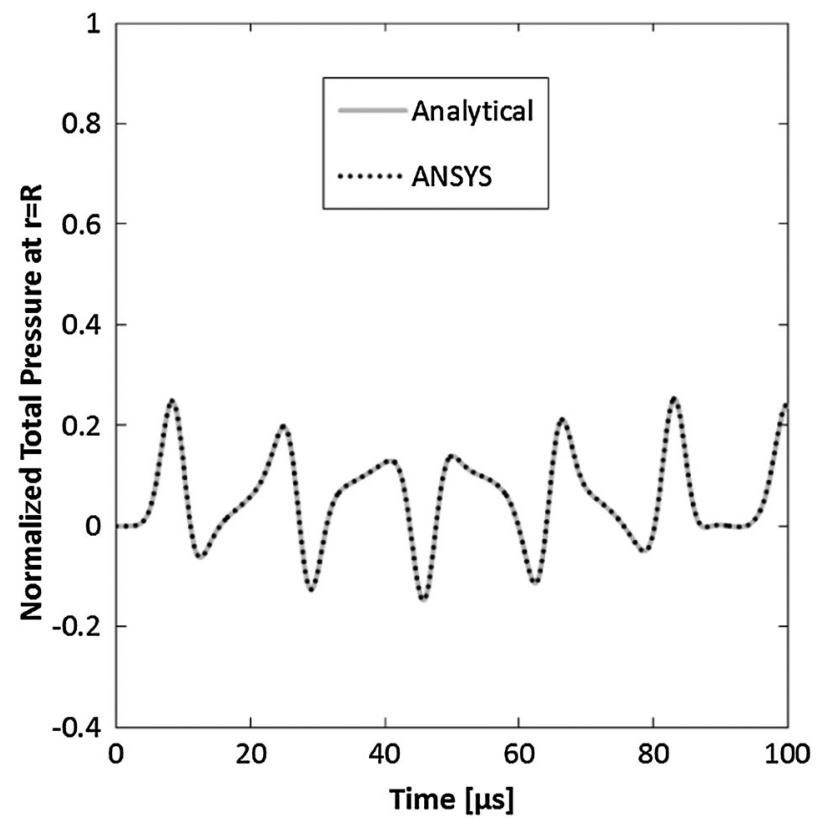

FIG. 6. Normalized total pressure at $r=R$ for the test case assuming plane stress.

achieve this agreement with the ANSYS simulation the courant number criteria must be respected i.e.,

$$
\delta t<\frac{\delta x}{c},
$$

where $\delta x$ is the size of a finite element cell and $\delta t$ is the maximum time step. In addition $\delta x$ must be small enough to resolve the peaks of pressure. The width of the pressure

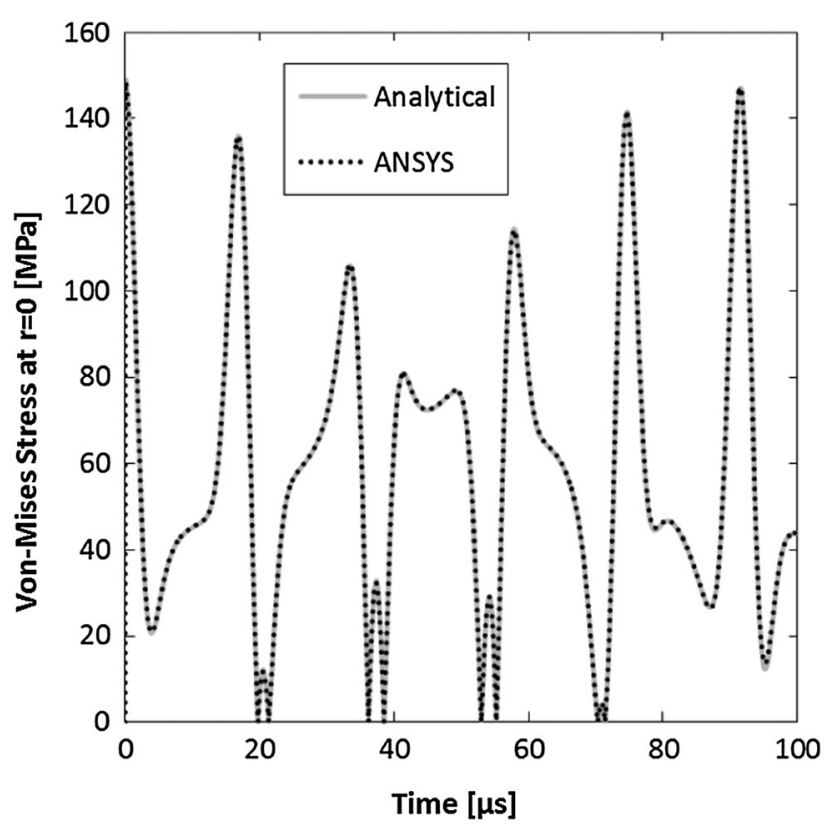

FIG. 7. Von-Mises stress at $r=0$ for the test case assuming plane stress. 


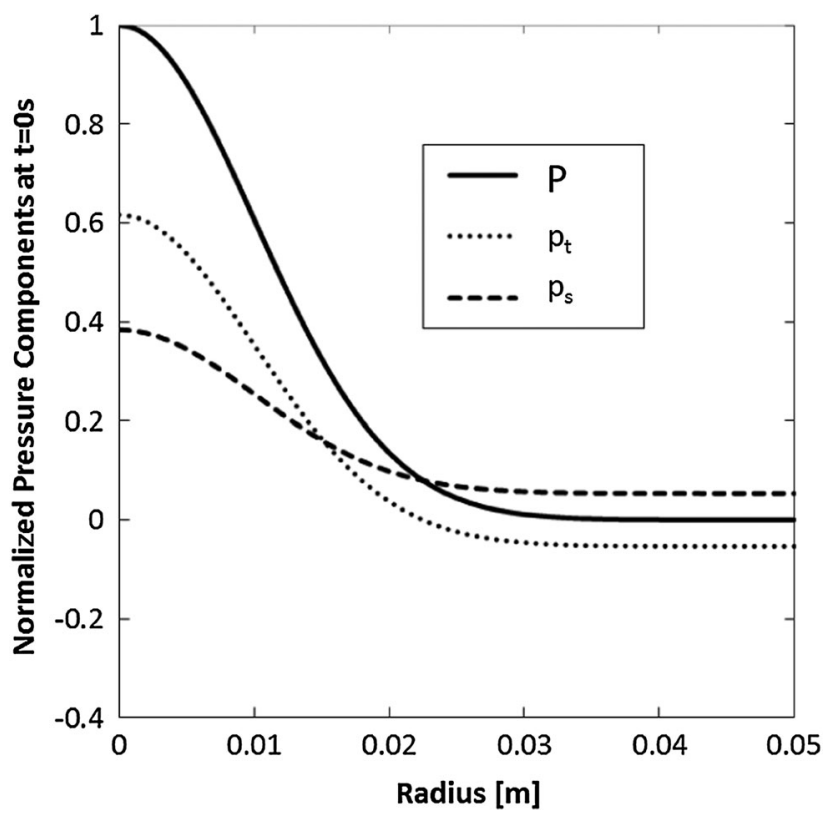

FIG. 8. Pressure wave components at $t=0$ for the test case assuming plane stress.

spikes depends on the sharpness of the initial temperature profile. A good guideline for a Gaussian initial condition is that

$$
\delta x<\frac{\sigma}{5}
$$

In order to capture the effect of instantaneous heating the initial time step in the simulation, during which the temperature rise occurs, must be sufficiently short.

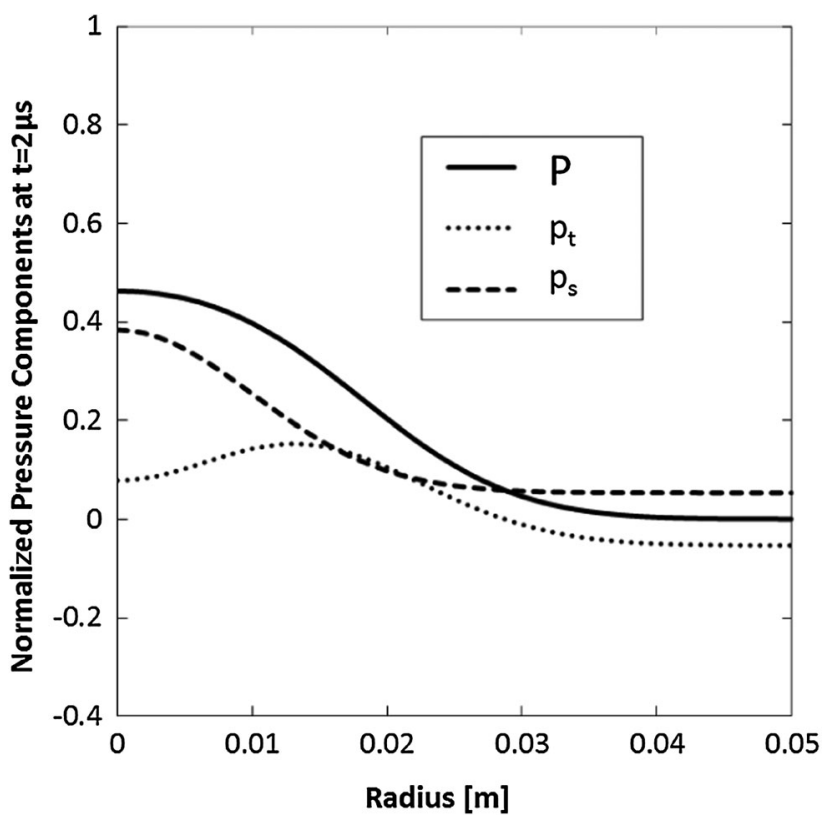

FIG. 9. Pressure wave components at $t=2 \mu \mathrm{s}$ for the test case assuming plane stress.

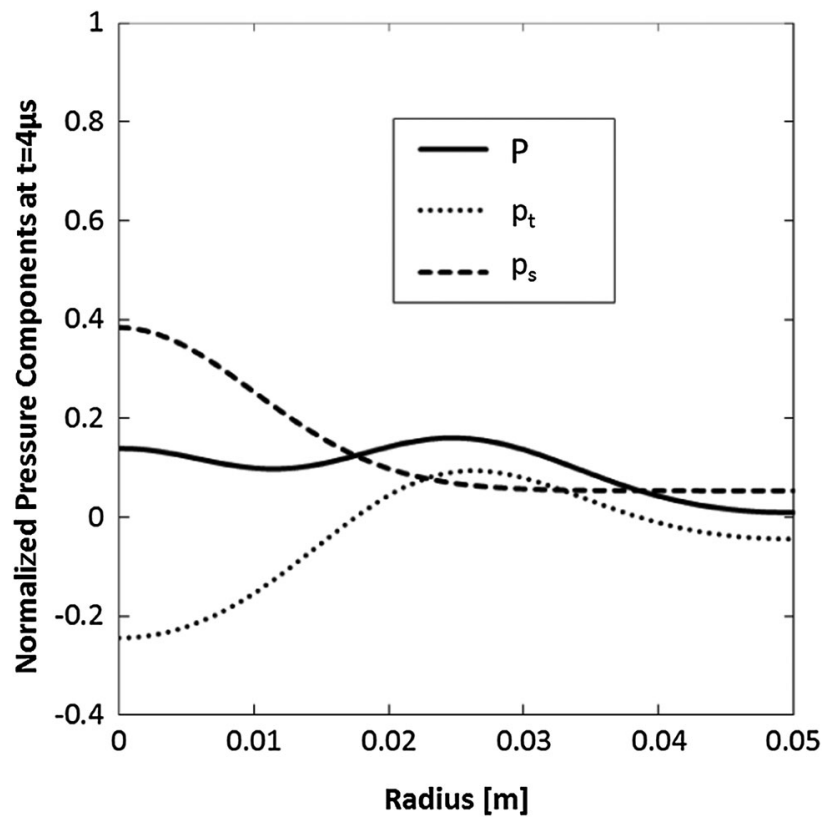

FIG. 10. Pressure wave components at $t=4 \mu$ s for the test case assuming plane stress.

Typically an initial time step that is 100 times shorter than the characteristic expansion time of the component in question will be sufficient, i.e.,

$$
\delta t_{1}<\frac{L}{100 c}
$$

where $\delta t_{1}$ is the initial time step and $\mathrm{L}$ is the characteristic dimension. This is easily tested by comparing the pressure

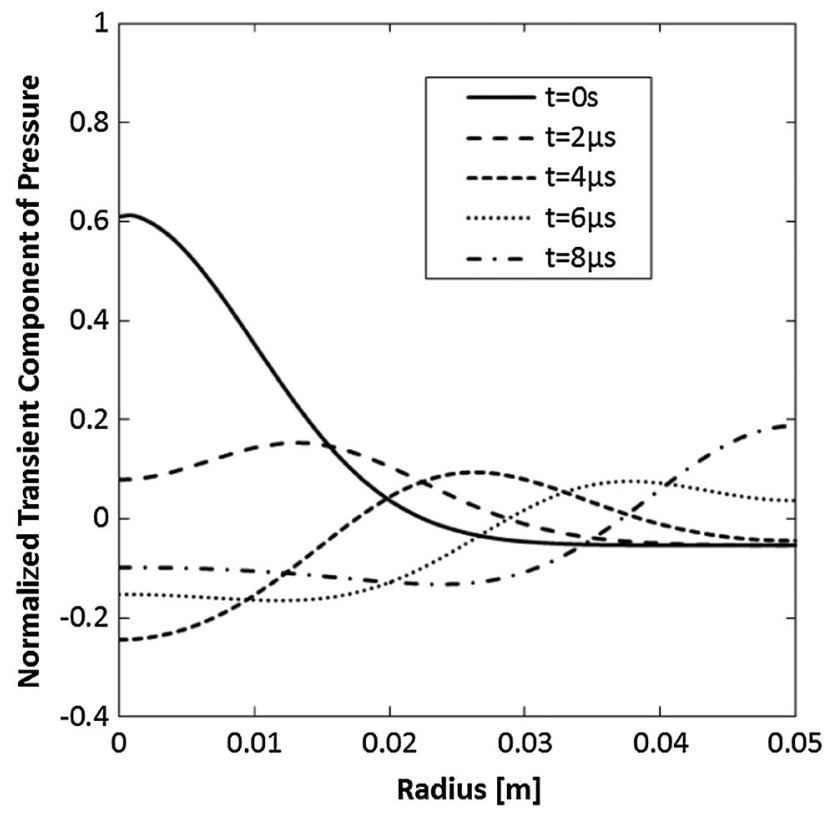

FIG. 11. Propagation of transient component of pressure for the test case assuming plane stress. 


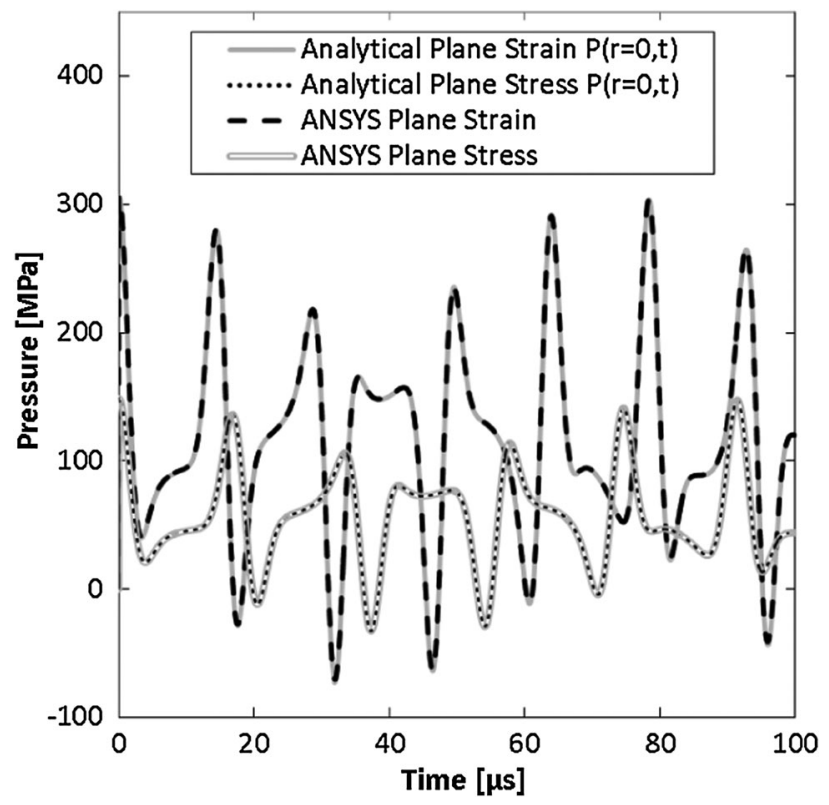

FIG. 12. Comparison of plane stress model representing a foil or window and plane strain representing a long cylinder.

at the end of the first time step to Eq. (22) for plane stress or (28) for plane strain.

To illustrate the hypothesis of pressure waves being decomposable into two components each component is plotted as a function of radius for several different times in Figs. 8, 9, and 10. Figure 11 shows the propagation of transient pressure resulting from Eq. (24). The final comparison shows the difference between a plane strain and plane stress model (Fig. 12). Again there is excellent agreement with the analytical solutions. It can be noted that the plane strain model exhibits higher pressure levels as a result of the axial constraint and that the fundamental oscillation frequency is higher due to the greater sound speed [Eq. (27) compared to Eq. (13)].

\section{CONCLUSIONS}

An analytical expression is formulated for the pressure waves induced by instantaneous heating of a beam window. The total pressure waves are shown to be comprised of a static and transient component. The analytical solution is shown to be bounded and stable. Solutions are presented for a plane stress model representing a foil or window and a plane strain model representing a long cylinder. Perfect agreement between the analytical solution and an implicit finite element method is achieved. Guidelines for achieving accurate calculation of pressure waves with finite element analysis are presented. These are principally that numerical time steps must be small enough to capture the passage of a wave as defined by the Courant number and that there must be enough spatial discretization to resolve gradients in the initial condition.

[1] P. Montanez and P. Sievers, Validation of a computer code for use in the mechanical design of spallation neutron targets, J. Neutron Res. 9, 1 (2001).

[2] G. P. Skoro, J. R. J. Bennett, and T. R. Edgecock, Yield strength of molybdenum, tantalum and tungsten at high strain rates and very high temperatures, J. Nucl. Mater. 426, 45 (2012).

[3] P. Sievers, Elastic Stress Waves in Matter due to Rapid Heating by an Intense High-Energy Particle Beam (CERN-LabII-BT-74-2, Geneva, 1974).

[4] J. Zheng, P. Xu, Q. Fu, R. Taleyarkhan, and S. Kim, Elastic stress waves of cylindrical rods subjected to rapid energy deposition, Proc. Inst. Mech. Eng. Part C 218, 359 (2004).

[5] R. Feynman, R. Leighton, and M. Sands, Lectures in Physics (Caltech, Pasadena, 2013), Vol. 1, Ch. 47.

[6] S. Timoshenko, Theory of Elasticity (McGraw-Hill, New York, 1951).

[7] H. Kolsky, Stress Waves in Solids (Dover, New York, 1952).

[8] A. Polyanin, Handbook of Linear Partial Differential Equations for Engineers and Scientists (Chapman and Hall/CRC, London, 2002).

[9] O. Adeyemi, Linear Acoustic Waves Induced in a Cylindrical Solid Target by Particle Beam in e--e+ Colliders (Hamburg University, Hamburg Germany, 2015).

[10] ANSYS $^{\circledR}$ Mechanical Enterprise, Release 16.1, ANSYS, Inc. 